\title{
Análisis de narrativas de los y las estudiantes con bajo rendimiento académico de algunas instituciones de educación secundaria del departamento de Antioquia en el año $2013^{*}$
}

\section{Analysis of low academic performance students' narrations from some secondary education institutions in Antioquia in 2013}

\author{
Diana Cecilia Pérez Posada* \\ Fray Durley Arrieta Osorio \\ Jesús Quintana Castillo \\ David Alberto Londoño Vásquez
}

Recibido 28. 04. 2016 • Arbitrado 20. 05. 2016 •Aprobado 15.06. 2016

\section{Resumen}

En este artículo se analizaron las creencias de los y las adolescentes que se encuentran con bajo rendimiento académico y las de las personas significativas de su entorno familiar sobre el estudio. Para lograrlo, se identificaron las creencias halladas en las narraciones de los y las adolescentes en las encuestas sociodemográficas y entrevistas realiza-

Es un artículo resultado de la investigación Relación entre las estructuras de creencias de los y las adolescentes con bajo rendimiento académico y con las de sus personas significativas, la cual se realizó para optar al título de Magíster en Educación y Desarrollo Humano del Cinde-Universidad de Manizales. Dicha investigación fue aprobada por los pares evaluadores en 2015-II.

** Magíster en Educación y Desarrollo Humano del Cinde-Universidad de Manizales y Licenciada en Educación Básica con Enfasis en Ciencias Sociales de la Universidad de Antioquia. Docente de la Institución Educativa Octavio Calderón Mejía. E-mail: dianac42@gmail.com

*** Magíster en Educación y Desarrollo Humano del Cinde-Universidad de Manizales y Psicólogo de la Universidad de Antioquia. Docente orientador de la Institución Educativa Playa Rica. E-mail: frayarrieta@gmail.com

***** Magíster en Educación y Desarrollo Humano del Cinde-Universidad de Manizales y Psicólogo de la Universidad de Antioquia. Docente de la Institución Educativa La Danta. E-mail: jesus8212@ hotmail.com

***** Doctor en Ciencias Sociales, Niñez y Juventud, investigador de la línea Estudios Éticos, Estéticos y Comunicativos del grupo de investigación en Psicologia Aplicada y Sociedad de la Institución Universitaria de Envigado, Docente de Tiempo Completo de la Institución Universitaria de Envigado. Researcher ID: F-8907-2013. ORCID: 0000-0003-1110-7930. Email: dalondono@correo.iue.edu.co 
das a las personas significativas del entorno familiar; con este insumo se describieron tales, con el fin de relacionar la información recolectada, así analizar si dichas creencias eran compartidas e influían en el proceso académico de los y las adolescentes, y si existían semejanzas o diferencias entre los participantes. El estudio se desarrolló a través de la metodología de la investigación cualitativa de carácter descriptivo-comparativo; se eligió como método investigativo el género narrativo propuesto por Leonor Arfuch. Las técnicas que se utilizaron fueron tres talleres escriturales dirigidos: la línea de tiempo escolar, las imágenes y la redacción de un cuento. Estas actividades se realizaron con consentimiento informado de los representantes legales de los y las adolescentes, dado que eran menores de edad. Finalmente, en los hallazgos de la investigación surgieron las siguientes categorías: los sentimientos, las actitudes, la sanción, la socialización, el rendimiento académico y las presunciones. De igual forma, se vislumbró una fuerte relación entre las creencias y el bajo rendimiento académico.

Palabras clave: Adolescentes, narrativas, creencias, rendimiento académico

\section{Abstract}

In this article low academic performance adolescence's beliefs and the meaningful people in their family environment's thought about study were analyzed. Therefore, the beliefs found in adolescences' narrations and meaningful people in their family environment's sociodemographic surveys and interviews were identified; with something like this input described such as relate the information collete and analyzed if this beliefs were sahred and influenced their academic procces and there were any similarities or differences between participants. The study was carried out by means of the qualitative research methodology, descriptive-comparative; the research method was the narration genre proposed by Leonor Arfuch. The chosen techniques were three written workshops: School time line, imagines and storytelling. An informed consent were signed out by their legal representatives in 
order to carry out these activities because the participants were under eighteen. Finally, in the research findings next categories emerged: feelings, attitudes, fines, socialization, academic performance and presumptions. Likewise, a strong relation between the beliefs and low academic performance were surmised.

Key words: Adolescences, narrations, beliefs, academic performance

\section{Resumo}

Este artigo foram analisados as crenças dos adolescentes que se encontram com baixo rendimento escolar e as pessoas significativas em seu ambiente familiar no estudo. Para conseguir isso, foram identificados as crenças encontradas nas narrativas dos adolescentes e os levantamentos sociodemográfico e entrevistas com as pessoas importantes de ambiente de família; com esta entrada, foram descritos narrativas, levantamentos demográficos e entrevistas, a fim de relacionar a informação recolhida, para asim analisar se tais crenças foram partilhados e influenciou os adolescentes processo acadêmico, e se havia semelhanças ou diferenças entre os participantes. O estudo foi realizado através de metodologia de pesquisa qualitativa natureza descritiva-comparativa; foi escolhido como método de investigação a narrativa gênero proposto por Leonor Arfuch. As técnicas utilizadas foram três trabalhos escritos dirigidos: linha de tempo escola, as imagens e escrever uma história. Essas atividades foram realizadas com consentimento legal informadas pelos representantes dos adolescentes, uma vez que eles eram menores de idade. Finalmente, sobre as conclusões da pesquisa do inquérito surgiram as categorias: sentimentos, atitudes, punição, socialização, desempenho acadêmico e premissas. Da mesma forma se vê, uma forte relação entre as crenças e baixo rendimento acadêmico.

Palavras-chave: adolescentes, crenças, narrativa, desempenho acadêmico 


\section{Introducción}

El bajo rendimiento académico es una situación que se ha presentado a través de los tiempos; sin embargo, en los últimos años, la problemática social y familiar conlleva a que se haga más profundo y que sean varias las causas de dicha situación, como son las dificultades de aprendizaje, la violencia intraurbana, la desmotivación escolar, las dificultades familiares, los bajo niveles de literacidad, ${ }^{1}$ entre otras.

Múltiples estudios señalan diversas causas; Bricklin y Bricklin (1988, citados en Sánchez Escobedo, 2011) refieren que las causas del rendimiento escolar insuficiente pueden clasificarse en cuatro categorías: biológico, pedagógicos, sociológicos y actitudes emocionales conflictivas. Otras investigaciones, como la realizada por la Secretaria de Educación de Bogotá en el 2010, "Estudio sobre los factores que influyen en el rendimiento escolar", explica el desempeño escolar a partir de factores endógenos y exógenos. Los factores endógenos hacen alusión a factores biológicos o psicológicos propios de cada persona, mientras que los factores exógenos hacen referencia a factores sociales, pedagógicos y ambientales.

Por otro lado, el bajo rendimiento académico se asocia en muchos casos al escaso acompañamiento y el poco compromiso que demuestran los acudientes de los y las estudiantes frente a los llamados de los y las docentes de la institución en situaciones de dificultades académicas, ya que en la mayoría de los casos no asisten, justificando tener otros compromisos como los laborales, por ello se escuchan las siguientes expresiones: "No tenemos tiempo para ir a la escuela y comprometernos [...] iTrabajamos todo el día!. O también "En mi casa dicen que la escuela es asunto de los profesores y no de los padres". ${ }^{2}$

La literacidad es un concepto propuesto por Cassany (2006), donde señala que esta "abarca todo lo relacionado con el uso del alfabeto: desde la correspondencia entre sonido y letra hasta las capacidades de razonamiento asociadas a la escritura" (p.38). Al respecto, Londoño (2015) amplía que "la literacidad requiere del desarrollo de competencias discursivas que estén relacionadas con el análisis del discurso y la argumentación" (p.92).

2 Expresionestomadas el 20 deenero de 2014 en:http://escueladecrianzas.blogspot.com/2008/03/ eva-gibertimaestra-de-maestros-dice.html 
En este mismo sentido, se identifica que algunos y algunas adolescentes no cuentan con el apoyo suficiente y el acompañamiento adecuado de las personas significativas en la realización de las tareas; puesto que como docentes, al revisar las actividades extraescolares encontramos que estas no fueron realizadas o elaboradas adecuadamente. Esto, en ocasiones, se materializa en los boletines periódicos del adolescente poniendo en riesgo la promoción del estudiante al grado siguiente.

Asimismo, en el bajo rendimiento académico, se resalta el proceso transitorio biopsicosocial en el que se encuentra el y la adolescente, ya que se concibe como un "periodo evolutivo de transición entre la infancia y la etapa adulta, que implica cambios biológicos, cognitivos y socio-emocionales" (Santrock, 2004, p.14). En este periodo transitorio biopsicosocial, el y la adolescente pasa de la educación básica primaria a la básica secundaria y es en este cambio, donde los y las adolescentes atraviesan por un estado de estrés, al evidenciar una serie de cambios en lo individual, familiar y escolar. Dichos cambios obedecen a...

...la pubertad y las preocupaciones relacionadas con la imagen corporal; la aparición de, al menos, algunos aspectos del pensamiento operacional formal, incluyendo los consecuentes cambios que se producen en la cognición social; una mayor responsabilidad e independencia junto con una menor dependencia de los padres; el paso de una estructura limitada al aula a un sistema escolar más grande e impersonal; el cambio de tener un profesor a tener muchos y de un grupo homogéneo y estable de compañeros a otro más heterogéneo; y la existencia de un mayor énfasis en los logros, los resultados y en su evaluación. (Santrock, 2006, p.415-416)

Lo anterior propicia que el y la adolescente en muchos casos no obtenga buenos resultados académicos y que su experiencia en el paso de la primaria a la secundaria no sea la más satisfactoria al tener que configurar un nuevo mundo escolar.

A través de la investigación, se procuró indagar y comprender si el bajo rendimiento académico que presentaban algunos y algunas adolescentes obedecía a las estructuras de creencias de los mismos, afectando su desempeño académico al no responder adecuadamente frente a los requisitos exigidos por la institución educativa. En otras palabras, si las estructuras de creencias infundados en el proceso de interacción 
familiar, social y cultural impactaban positiva o negativamente el desarrollo de las actividades académicas de los y las adolescentes.

\section{Las creencias}

Para Van Dijk (200o, pp.36-37), las creencias son:

...todos los productos del pensar serán declarados como creencias. En otras palabras, las creencias son los ladrillos del edificio de la mente. El conocimiento en este caso, es solamente una categoría específica de creencias, a saber, aquellas creencias que "nosotros" (como grupo, comunidad, cultura, caso particular o institución) consideramos "creencias verdaderas" de acuerdo con ciertos fundamentos o criterios de (verdad). Estos criterios establecen que las creencias (para nosotros) son válidas, correctas, certificadas, sostenidas de una manera general o que reúnen los estándares de verdad socialmente compartidos (Van Dijk, 2000, p.35).

Esta propuesta conceptual nos permitió, a través de las narrativas, develar algunas creencias que los y las adolescentes participantes han construido durante sus vidas. Puesto que estas construcciones pueden verse reflejadas en las prácticas discursivas, ${ }^{3}$ entendiendo que el lenguaje es una construcción socio-cognitiva y de la cual las narrativas hacen parte esencial.

Es importante mencionar que estas construcciones se han realizado bajo criterios sociales, culturales e históricamente variables.

En este sentido las creencias no son solamente productos subjetivos sino también objetivos del pensamiento o incluso infundados o contrarios a la verdad, o creencias (como las religiosas) que son aceptadas como las verdaderas por un grupo específico de personas, sino que también incluyen lo que llamamos conocimiento (Van Dijk 2000, p.36).

Finalmente, las creencias no son pensamientos que se limitan a lo que existe, a lo que es o puede ser verdadero o falso. Sino que también

3 Entiéndase como práctica discursiva, "Un conjunto de reglas, históricas, siempre determinadas en el tiempo y el espacio que han definido en una época dada y para una área social económica, geográfica o lingüística dada, las condiciones de ejercicio de la función enunciativa” (Foucault, 1991, p.198). 
pueden corresponder a evaluaciones, o sea, a lo que nosotros pensamos, encontramos que es verdadero o falso, agradable o desagradable, permitido o prohibido, aceptable o inaceptable, es decir, a los productos de juicios basados en valores o normas. En otras palabras, que las creencias están constantemente en la posibilidad de ser confrontadas con la realidad y cotidianidad del individuo.

\section{Metodología}

Tipo de investigación

El estudio se desarrolló a través de la metodología de la investigación cualitativa de carácter descriptivo-comparativo, ya que se abordaron las realidades subjetivas e intersubjetivas de los y las adolescentes y las personas más significativas del entorno familiar, conociendo y comprendiendo las lógicas de pensamiento que guía las valoraciones que se tejen en torno al estudio.

\section{Método investigativo}

Con el propósito de desarrollar los objetivos propuestos en la investigación, se eligió como método investigativo el género narrativo, el cual nos permitió interpretar y analizar los narrativas de los y las adolescentes que presentaban bajo rendimiento académico del grado sexto de tres instituciones educativas del departamento de Antioquia y si las narrativas podrían estar relacionadas con las significaciones colectivas construidas a través de las estructuras de creencias, procuradas en las prácticas comunicativas por las personas significativas del entorno familiar a los y las adolescentes.

Por consiguiente, para sistematizar las narrativas de los y las adolescentes y las encuestas y entrevistas de las personas significativas de su entorno familiar, la investigación se apoyó en la propuesta de Leonor Arfuch, sobre el género narrativo, donde la autora resalta la narrativa como “...algo más que la simple configuración de relatos; [sino como]... un vehículo para la comprensión e interpretación de las personificaciones, de las relaciones entre los sujetos y de sentidos contextualizados en el tiempo y el espacio" (Arfuch, 1995, p. s.p). Con el género narrativo 
se pretendió desentrañar los sentidos que trasmitían las personas significativas del entorno familiar por medio de las estructuras de creencias a los y las adolescentes sobre la relevancia del estudio.

\section{Técnicas o instrumentos para la recolección de la información}

Las técnicas que se utilizaron para dar cuenta de las narrativas de los y las adolescentes, fueron tres talleres escriturales dirigidos a partir de las siguientes estrategias: la línea de tiempo escolar, donde los y las adolescentes relataron su experiencia escolar a través de la línea de tiempo; las imágenes, en esta estrategias ellos y ellas realizaron una descripción sobre lo que veían y lo asociaron a sus vidas; y la redacción de un cuento, donde ellos y ellas escribieron sobre la importancia de la escuela y la familia.

Cabe aclarar que esta actividad con los y las participantes, se realizaron con el consentimiento informado de sus representantes legales, dado que eran menores de edad, y por ello, se constituían como personas vulnerables y por medio del acta se les protegería sus intereses y derechos.

Por otro lado, a la persona significativa del entorno familiar que fue seleccionada por cada uno de los y las participantes, se le aplicó una encuesta sociodemográfica, la cual dio a conocer el contexto familiar y social. Ella se empleó “como un método de obtención de información mediante preguntas orales o escritas, planteadas a un universo o muestra de personas que tienen las características requeridas por el problema de investigación” (Briones, 2002, p.52). Así, se obtuvieron datos de la constitución familiar, cultural y social que luego fueron corroborados con una entrevista.

Con la entrevista como técnica del presente estudio, los investigadores se acercaron a las vidas de los otros y, a través de sus narrativas, identificaron, describieron y compararon aquellas estructuras de creencias sobre el estudio que construyeron los y las adolescentes en su medio, en el cual las personas significativas del entorno familiar jugaron un papel primordial. 
También con la entrevista se triangulo la información recolectada en las narrativas de los y las adolescentes y las encuestas sociodemográfica a las personas significativas del entorno familiar, analizando si las estructuras de creencias eran compartidas e influían en el proceso académico de los y las adolescentes.

Las narrativas que contendrán la descripción propia de los y las adolescentes fueron analizadas por los investigadores desde el referente conceptual de Teun Van Dijk.

Las tres instituciones educativas a las cuales pertenecen los y las participantes que formaron parte de la presente investigación tienen las siguientes características:

La institución educativa La Danta se encuentra en el corregimiento La Danta del municipio de Sonsón, los estratos socioeconómicos del corregimiento oscilan entre 1 y 2 , sin embargo, es importante dar a conocer que en la población se encuentran familias desplazadas por la violencia y desmovilizados. Se registra que los y las estudiantes de la institución pertenecen a familias monoparentales con jefatura femenina, compuestas, extensas y algunas son nucleares.

Por otro lado, la institución educativa Octavio Calderón Mejía ubicada en el barrio Campo Amor de la comuna 15 de Medellín, los estratos socioeconómicos del sector oscilan entre el 3 y 4; sin embargo, los estudiantes que alberga la institución pertenecen a los estratos 2, 3 y 4. Se registra que los y las estudiantes de la institución pertenecen a familias monoparentales con jefatura femenina, compuestas, extensas y algunas son nucleares.

Y la institución educativa Playa Rica ubicada en el municipio de Bello, los estratos socioeconómicos del sector oscilan entre el 1 y 3; no obstante, la gran mayoría son estrato 2, además, un gran número de la población son víctimas del desplazamiento. Los y las estudiantes en su gran mayoría pertenecen a familias recompuestas o familias monoparentales con jefatura materna.

Los y las participantes que fueron seleccionados para la realización de la investigación presentaron bajo rendimiento académico y 
estaban cursando nuevamente el grado sexto, se establecieron 2 participantes por cada institución educativa, es decir, 6 adolescentes en total y aproximadamente 6 personas significativas del entorno familiar de los mismos, es decir, 1 por cada adolescente, el cual fue seleccionado de acuerdo a la opinión del mismo y a los resultados que arrojaron las narrativas.

\section{Resultados}

$\mathrm{Al}$ identificar, describir y contrastar las diferentes narrativas de los y las adolescentes participantes, y las encuestas sociodemográficas y las entrevistas de las personas significativas, se encuentran en las narraciones algunas características que predominan y que son puntos de encuentro entre los y las diferentes participantes. Por tal razón, surgen una serie de categorías emergentes que dieron cuenta de las estructuras de creencias que tenían los y las participantes de la investigación sobre la importancia del estudio.

A continuación, se abordarán las siguientes categorías emergentes: sentimientos, vínculo, sanción, relaciones familiares, actitud, presunciones, socialización y rendimiento académico.

\section{Sentimientos}

Para Anna María Fernández Poncela (2011, p.3) los sentimientos "son las emociones culturalmente codificadas, personalmente nombradas y que duran en el tiempo. Secuelas profundas de placer o dolor que dejan las emociones en la mente y todo el organismo". Los sentimientos son más duraderos, permanecen más tiempo en la mente y por ello pueden ser nombrados e interpretados.

En las narrativas ${ }^{4}$ se logró identificar que algunos de los y las adolescentes reconocen en sus personas significativas un deseo de que ellos

4 Con el objetivo de hacer un análisis de las narrativas de los y las participantes, al interior del cuerpo del escrito por comodidad y agilidad del lector, solo se tomaran en cuenta fragmentos de las narrativas. Los fragmentos de narrativas se mantendrán conforme a los errores gramáticas, puntuación, acentuación y repeticiones que los y las participantes manifestaron. 
y ellas sean mejores personas, sujetos útiles a la sociedad y que, en ocasiones, esto implica para los cuidadores sobreesfuerzos y sacrificios en pro de poderles brindar el apoyo que permita la superación, estructurando creencias en los y las adolescentes; sin embargo, vale la pena resaltar el enunciado del participante $L:^{5}$

"A veces uno no sabe valorar lo que los padres hacen por uno..."

Cuando el participante hace mención de lo anterior, es probable que se esté refiriendo a expresiones tales como la manifestada por la persona significativa de $E$, "Uno briega a hacer lo que sea por ellos. O sea, a mí me ha tocado muy duro con $E$... [¿Duro es qué?] ...es que hasta sin trabajo y sin nada así he luchado por ella”.

Lo anterior, es un reflejo de la situación de $L$, ya que puede generar un posible sentimiento de culpa debido a su comportamiento, actitud y bajo desempeño académico, los cuales no corresponden a las expectativas que le manifiesta su persona significativa. Además, el participante revela sentimientos donde se tiene la obligación moral de corresponder al interés de los padres y las madres, acatar sus consejos, valorar sus palabras y cumplir la voluntad de ellos.

Por otro lado, está la forma como los padres y las madres alientan a este participante a continuar su proceso formativo escolar, a pesar de que él no deseaba asistir a sus clases, era mandado a la escuela, mostrando una dicotomía entre lo que deseaba y lo que le era impuesto.

En el proceso académico, el participante $L$ hace alusión a situaciones que lograron motivarle como lanzarse a la personería escolar y ser elegido, y ser promovido al siguiente grado, como lo hace saber en la siguiente narrativa, $(L, 2014)$ "En quinto me postulé para ser personero de la escuela y gané me sentí muy bien”. “...pase al tercer grado me dio mucha alegría porque había pasado el año”.

5 Los y las participantes de la presente investigación son seis adolescentes y seis personas significativas, las cuales serán nombradas a partir de las iniciales de su nombre, con el fin de conservar la integralidad y confidencialidad de los y las participantes. 
Fuera de lo anterior, vale la pena resaltar el énfasis que el participante $L$ hace sobre la culpa, cuando expresa que:
"A veces uno no sabe valorar lo que hacen los padres por uno pero más adelante vas a ver que sí tuviste que estudiar y valoraras todo lo que exigieron tus padres y para los que sí valoraron van a decir gracias a Dios que si estudié y gracias a mis padres hoy soy alguien muy importante en la vida y en la sociedad".

Esto revela que el adolescente ha introyectado una mirada adultocéntrica, puesto que socialmente se espera que la dedicación y entrega de los y las cuidadores sea retribuida por los y las adolescentes cuando los adultos y las adultas se encuentren en una etapa no productiva.

De igual manera, el participante $L$ continua brindando diferentes elementos que valen la pena detenerse a pensar, entre ellos hace alusión a su gusto por estudiar al iniciar su proceso formativo en preescolar, "cuando yo comencé a estudiar en preescolar me dio mucha alegría”, sin embargo, se logra identificar que a través de este proceso formativo se presentaron diferentes hechos que hace que haya una fractura por su gusto y alegría por continuar estudiando, perdiendo un interés más natural y genuino, pues en su narrativa comienza a nombrar el estudio como una obligación, como una carga asignada por el Otro "me entraron a la secundaria pero no quería estudiar", nótese que el participante al hacer dicha expresión da una connotación negativa por la obligatoriedad que resulta de la decisión tomada por el otro y no se tiene en cuenta su propia opinión.

Por otro lado, los sentimientos que surgen en las narrativas de los y las adolescentes con respecto a su vinculación a la escuela revelan un grado de felicidad por asistir al proceso formativo especialmente en el grado inicial.

Lo académico se circunscribe en la gran mayoría de los casos a lo cognitivo, ignorando en varias ocasiones los sentimientos, por la dicotomía preexistente entre razón y sentimientos, donde lo más valorado en estos espacios es la razón. Los sentimientos que se albergan en estos 
lugares son generados por el mismo entorno escolar o por sus personas significativas, los cuales pueden crear motivación o apatía hacia el estudio, lo que los vincula o desvincula de los compañeros, las compañeras, los y las docentes y sus personas significativas.

Después de exponer la categoría de sentimientos que emergió en las narrativas de los y las participantes, continuamos con la de vínculos.

\section{Vínculos}

Existe algo inherente al ser humano que hace que sea posible no solo entendimiento con el otro sino, además, que posibilita el desarrollo de nosotros mismos como personas, la conservación, el cuidado y es precisamente las relación afectivas que establecemos entre sí con las personas más cercanas; este tipo de relación también es conocida como vínculo.

Para John Bowlby (1969) el vínculo hace referencia "al lazo afectivo que emerge entre dos personas y que genera un marco de confianza en el otro y en la vida, en un contexto de comunicación y de desarrollo" (p.293).

Los y las participantes en sus narrativas mencionan diferentes personas que han acompañado su proceso formativo, atribuyéndoles algunas características que los constituye en personas significativas, estableciendo algún tipo de vínculo afectivo atravesado por la atención, el cuidado, la preocupación por el bienestar, el apoyo, el respaldo, entre otras.

En las narrativas de los y las participantes se identifica que sus personas significativas en su mayoría son las madres, como es el caso de $M A$ quien manifiesta, "Mi mamá es la persona más significativa que [he] tenido durante el proceso educativo porque ella me apoya"

De igual manera, se identifica que para algunos adolescentes la figura del docente ocupa un lugar especial en el proceso formativo del mismo, como también en un apoyo para sobrellevar las situaciones complejas en que estos se encuentran en determinados momentos o etapas de la vida escolar. Pues, encontraron, en ellos, características que les permitían establecer relaciones de confianza y cercanía. Tal como se puede observar en las siguientes narrativas: 
$E$ : "En tercero conocí a la profe Bibiana ella me enseño y me aclaro muchas cosas que no sabía así fue más fácil conocer lo que no sabía me gustaba mucho español porque era con ella y nos explicaba"

$L:$ “...mi mamá se puso a vivir con un señor que me maltrataba y una profesora que me quería mucho me ayudó con ese problema y lo mandaron para la cárcel y al tiempo la profesora se la llevaron para otro colegio y gracias a ella pase al tercer grado"

$K$ : "Un profesor que me haya marcado era una profesora que se llamaba Rosa, ella era tan buena, ella más que una profesora, yo la veía como una amiga, yo en ella confiaba más que en el psicólogo donde yo estaba, ella siempre estaba ahí, yo siempre le contaba las cosas, ella estaba ahí..."

$J E:$ "Pues yo diría que la profesora de Inglés porque fue de las mejores profesoras que yo tuve en ese tiempo, como con la que me llevaba más bien como la mejor"

Este tipo de relación establecida entre los y las participantes y sus docentes de acuerdo a su grado escolar también permite evidenciar que algunos de ellos no cuentan con el apoyo suficiente para realizar los trabajos académicos como lo son las actividades extraescolares y las tareas que son propuestos por la institución, ya que algunas de sus personas significativas sienten que no tienen la capacidad intelectual para ayudar a sus hijos e hijas, pues se autonombran de una forma especial que le dice directamente al participante que no se tiene la capacidad para hacerlo, como es el caso de la persona significativa de la participante $E$ : “Ah, qué pues no haber tenido estudio, soy como soy, bruta, maleducada”.

Por otro lado, se hace necesario aclarar que los y las participantes no cuentan con una única persona significativa, sino, que está sujeta al contexto y a los diferentes tiempos en los que se encuentra, además para efectos de esta investigación, se hizo principal énfasis en la persona más significativa de su entorno familiar.

Aquellas personas que se vinculan en sus roles de cuidadores, tienen como funciones socialmente asignadas instruir, corregir, cuidar, proteger, resguardar a aquellas personas que se encuentran bajo su encargo, y, la vez, sancionar cuando ellos de acuerdo a su criterio lo consideren necesario. 


\section{Sanción}

La sanción es otra de las categorías emergentes que surgieron de la investigación; la cual se constituye en un mecanismo de control, donde se regulan las acciones, pretendiendo que las personas se comporten conforme a lo esperado y estipulado por la norma. La sanción hace parte de la cotidianidad del ser humano porque su principal razón es mitigar los acontecimientos que están sucediendo en un determinado espacio y momento.

La sanción es manifestada por las personas significativas de los y las participantes de diversas formas, entre ellas la exhortación, como una advertencia de frenar acciones que no satisfacen los gustos que tienen dichas personas, como es expresado en la siguiente narrativa de $L$ : "Pero el otro año me entraron a sexto a estudiar y me dijeron que si perdía el año me mandaban a trabajar”, interpretándose la anterior narrativa como el deber de hacer las cosas bien, tal como lo desea su persona significativa o de lo contrario obtendrán un castigo por ello. Lo anterior es reforzado en la siguiente narrativa de $J E$ :

"Pues no, quitar ciertas cosas como la televisor, el internet, las salidas con los amigos, para ir demostrando ese castigo como tú no haces esto bien y esto te pasara pues porque es un pequeño... ummm... se me fue la palabra... reprendimiento".

Además, de la exhortación como la forma de anticipar, advertir al participante sobre lo que le pasará si no hace las cosas deseadas por su persona significativa. También aparece la sanción desde el castigo físico hacia los participantes, como lo menciona la siguiente participante:

E: "Mi mamá se portaba muy bien conmigo, aunque a veces que perdía las evaluaciones me pegaba y me regañaba y me castigaba con lo que a mí más me gustaba"

De igual modo, las personas significativas reconocen su necesidad de intervenir a partir de diferentes modos de ejercer autoridad, apli- 
cando sanciones que van desde una forma conciliadora como es el diálogo hasta el recurrir a estrategias tales como el castigo físico o la privación de ir a la escuela, este último con el afán que él o la adolescente genere una reflexión crítica en la que valore el estudio. Tal como lo evidencia la siguiente persona significativa:

Persona significativa de $K$ : “... La verdad yo la he castigado, le he pegado, he hablado, que no habré hecho con ella... que si no se pone pilas la saco de estudiar, y que la mando donde el papá, pero nada de eso funciona, hasta he pensado dejarla en la casa a ver si así piensa un poquito y valora el estudio".

Además, algunos de estos castigos físicos se realizaban en otros escenarios diferentes al hogar como expresa L: "Mi mamá me pegó delante mis compañeros". Dicho castigo se hace en público llevado a cabo por la madre, el cual se puede interpretar como un cambio de escenario pasando de la intimidad a lo pública; con este la madre busca generar cambios sobre la actitud que el adolescente tiene frente al estudio.

De igual forma, se logra evidenciar que las instituciones educativas realizaron sanciones hacia los participantes por no cumplir o basarse en los lineamientos establecidos por el manual de convivencia como lo nombra $L$ : "lo aporreé se rompió la cabeza y me impulsaron [expulsaron]".

Como se menciona anteriormente, las sanciones no corresponden únicamente al escenario familiar, pero es este espacio donde toman más fuerza al ser suministrados por las personas significativas de los y las adolescentes, incidiendo así en las diferentes formas de relacionarse con las personas que integran su núcleo familiar.

\section{Relaciones Familiares}

Las relaciones familiares surgen como otra categoría emergente en las narraciones de los y las adolescentes, y en las encuestas sociodemográficas y entrevistas de las personas significativas.

Las relaciones familiares suelen ser una rica fuente de apoyo para sus miembros en general, especialmente para los hijos e hijas, ya que 
ellos dependen en gran medida y necesitan del acompañamiento constante de sus padres y madres con el fin de velar, contribuir en el desarrollo y proceso formativo de los mismos.

Tener buenas relaciones familiares aporta a la calidad de vida, generando bienestar, brindando seguridad y una mayor tranquilidad, además, promueve la participación e interacción con todos aquellos que la integran y esto no solo se manifiesta en el entorno familiar, sino además, irradia los otros campos del ser humano como el ambiente escolar, social y laboral.

Sin embargo, cuando las relaciones familiares son conflictivas entre sus miembros, estas afectan significativamente los ambientes o contextos en los cuales participa el individuo. Es decir, las relaciones familiares inciden preponderantemente en los diferentes comportamientos que una persona tiene en su cotidianidad.

En diferentes estudios como el de Hernando, Oliva y Pertegal (2012) se menciona cómo los estilos parentales influyen en el rendimiento académico del adolescente; por otro lado, Rossas Sosa (1997) analizó la influencia del funcionamiento de la familia en el rendimiento académico del adolescente, hallando en sus resultados que en las familias con un nivel de funcionamiento familiar ${ }^{6}$ balanceado es más probable que los miembros tengan éxito.

De igual modo, Tilano, Henao y Restrepo (2009) proponen que "los estilos de autoridad inductivos favorecen el desempeño académico, mientras que los rígidos propician un desempeño académico deficitario, siendo más bajo en los permisivos" (p.1). Con lo anterior se valida la percepción que se tiene acerca del rendimiento académico en relación a los estilos parentales, ya que se evidencia que cuando hay autoridad en los hogares, esto genera impactos favorables en el rendimiento académico de los y las adolescentes, a diferencia de los estilos permisivos y rígidos, donde los resultados académicos son desfavorables.

6 El nivel de funcionamiento familiar es el resultado que se obtiene al valorar las dimensiones de cohesión y de adaptabilidad del Modelo Circunflejo de Olson (1983), clasificando las familias en: nivel balanceado, nivel medio y nivel extremo. 
Finalmente, las narrativas de los y las adolescentes exponen que las relaciones familiares se tornan en algunas situaciones difíciles, afectándoles en muchas ocasiones su autoconcepto y autoestima, evidenciándose esto en su actitud hacia el estudio.

\section{Actitud}

Una de las categorías emergentes fue el de la actitud, esta categoría alude a la posición que un sujeto asume frente a algo, es una postura que se elige en determinados momentos o situaciones de la vida cotidiana. Dentro de las manifestaciones de actitud se logra identificar en las narrativas de los y las participantes un concepto que ellos nombran "pereza" como la negligencia, falta de ganas o descuido en la realización de las obligaciones, especialmente referido al entorno escolar, incidiendo por ende en su percepción del estudio y el rendimiento académico de los y las estudiantes. Como dice el participante $L$ : “...me entraron a la secundaria pero no quería estudiar y no hacia las tareas, era muy irresponsable pero después quise ganar el año pero no fui capaz y lo perdí”.

Dicha narrativa manifiesta que sus obligaciones son establecidas por el otro, donde carecen de motivación propia. Es decir, que el participante siente pereza al estudiar porque no se siente convocado, atraído por lo que se le plantea, quizá lo siente como algo que no servirá, no contribuye en su desarrollo o carece de practicidad para la vida, lo cual hace que el cumplimiento de los deberes exigidos presente dificultades de realización y entrega.

La narrativa de L: "Mi mamá me pegó delante de mis compañeros y yo le cogí mucha pereza al estudio" aparece como un suceso, confirmando que existen acontecimientos que marcan la posición que un sujeto asume hacia determinados procesos o eventos; produciéndole el sentimiento de no querer volver a la escuela por lo que su madre le hizo delante de sus compañeros: vergüenza, rabia o enojo.

Otra de las narrativas que revelan una actitud que el participante $L$ toma frente al estudio es: "con el tiempo me fue dando pereza es- 
tudiar", se manifiesta como aquellos sucesos o acontecimientos que ocurren en la vida del participante que van generando desmotivación o reacciones de no querer seguir estudiando, sintiendo quizá este como una forma de castigo, pues volver a la institución es como seguir presenciando momentos que no le son agradables o satisfactorios a él, como lo confirma la siguiente expresión de $L$ : "yo no hacía nada, sólo desorden y mantenía peleando con los demás compañeros”. Y estos comportamientos que suenan como hechos reiterativos realizados por el participante puedan afectar la relación con su entorno familiar, ya que este podría significar castigo por parte de su persona significativa hacia él, como lo dice $L$ : "lo aporree, se rompió la cabeza y me expulsaron y mi mama[á] me pegó", además de los castigos realizados por la institución educativa, pues en contadas ocasiones dicho participante fue expulsado de la institución.

Este tipo de comportamiento nos hace pensar que la actitud que el participante nombra como pereza es estimulada en lo posible por el entorno, contexto en el cual se encuentra.

La actitud que asume cada uno de los y las participantes en relación con la responsabilidad frente al estudio tiene que ver con el rendimiento académico que tiene cada uno de ellos y ellas, es decir, cuando es un asunto más conciliado que obligado, escogido desde la libertad más que desde la imposición, presentaría probablemente mejores resultados.

Igualmente, como el participante $L$ expresa "ahora estoy otra vez en sexto pero también voy mal, me relaje”, hace muestra que no existe una motivación que lo impulse a cumplir con las exigencias académicas, pues no se siente convocado, "Con el tiempo me dio pereza estudiar", esta expresión del participante $L$ denota que algo paso en su actitud frente al estudio, se sospecharía que tiene relación a las sanciones las cuales enuncia debido a sus continuas peleas en el colegio.

Por otra parte, el participante $M A$ introduce la rebeldía como una actitud que asumió, responsabilizando las compañías y amistades, como el mismo lo dice "yo tome la actitud de rebeldía hacia el estudio por las amistades", esto lo condujo a prestar mayor atención a otros 
temas, descuidando el estudio a pesar de las observaciones continuas de sus padres.

También, en esta categoría hay una muestra de los intereses de los y las adolescentes, de cómo en esa búsqueda de independencia presentan gustos por compartir con sus pares, estar en un espacio diferente a la casa o como la participante $A M$ misma nombra: "Yo considero que me ha ido mal en el estudio es porque prefiero andar la calle que hacer las tareas”. La participante $K$ expresa que "me gusta estudiar"; sin embargo, nos asalta la duda si es por el hecho de asistir y aprender de las diferentes áreas o es un interés de compartir con el otro, con sus pares en un espacio de socialización como lo es la escuela, de asistir al colegio para encontrarse con sus amigos y amigas o con los compañeros y las compañeras.

La actitud de los y las adolescentes no es coherente con otras creencias que ellos y ellas están reproduciendo en sus narrativas, ya que lo que mencionan constantemente es una actitud de apatía y pereza frente al estudio, actitud que no les permite en este momento materializar la creencia del estudio como vehículo para mejorar su calidad de vida.

Algo similar se identifica en la narrativa de las personas significativas, pues algunas de ellas muestran incongruencia entre lo que piensan y quieren siendo contradictorio con sus propias experiencias de vida, tal como se evidencia en la siguiente narrativa, cuando se le pregunta acerca de su propia posibilidad de estudiar:

Investigador: ¿̇Te gustaría estudiar?

Persona significativa de E: No, ya no...

Investigador: ¿Por qué?

Persona significativa de E: Porque no. No... no me gusta, sigo con mi brutalidad"

Además, algunas de las personas significativas argumentan varias razones por las cuales no iniciarían o continuarían con su estudio, tales como: a) falta de tiempo (trabajo, cuidado de los hijos, tareas del hogar), b) la edad, c) tiempo transcurrido entre el momento actual y el 
último año estudiado, d) falta de oferta educativa teniendo en cuenta la jornada (nocturna), e) todo lo anterior se sustenta en las siguientes enunciados de las narrativas: ${ }^{7}$

Persona significativa de $K$ : "A mi si me gustaría mucho (estudiar), pero uno ya tan viejo pa' que, yo ya creo que a mí se me olvido todo, y además con que tiempo por Dios, a uno ya no le da tiempo sino para el trabajo y la casa".

Persona significativa de $L$ : "Si, si he pensado (estudiar) sino que las veces pues que me ha llegado, siempre he tenido pues trabajo que me ocupan como mucho y también los niños, siempre he tenido como niños pequeños y como que no se me ha presentado como la oportunidad como de estudiar"... "si fuera nocturna porque de día me queda prácticamente muy imposible, porque uno tiene que trabajar y atender los hijos y lo de la casa. Pero en nocturna sí, si a mí se me presentara la oportunidad de estudiar nocturna yo estudiaría”

Se observa cómo algunas de las personas significativas manifiestan interés por iniciar o continuar con sus estudios académicos, no obstante, no logran materializarlo pues presentan otras prioridades, que para ellas les impide seguir con su formación académica.

Por lo contrario, se encuentran que otras de estas personas significativas a pesar de sus ocupaciones, consideran importante el estudio pero a diferencia de las anteriores, llevan a cabo su proceso formativo porque lo consideran necesario para mejorar su calidad de vida y la de su familia, tal como lo menciona la persona significativa de $J E$ : "Yo me encuentro estudiando actualmente una tecnología en gestión de proyectos en el Sena"

7 Entiéndase por narrativas cada intento que hace el participante por narrar su ser. Es decir, poner en términos lingüísticos, lo real y lo irreal que constituye el ser del participante. No hay tiempos, no hay lógicas, pero a través del lenguaje se develan situaciones, pensamientos y creencias. Los relatos constituyen las narrativas, y a su vez, los relatos están compuestos por enunciados. Sería muy oportuno que tuviéramos eso claro a la hora de hacer referencia a las unidades de análisis. 


\section{Presunciones $^{8}$}

Otra de las categorías emergentes que surgieron de la investigación fue las presunciones, ellas al igual que las creencias son "productos o propiedades del pensamiento y, por lo tanto, también están asociadas con la mente. Aquí se entiende por creencia cualquier cosa que puede ser pensada" (Van Dijk, 2000, p.35), un estado de la mente en el que un individuo tiene como verdadero el conocimiento o la experiencia que tiene acerca de un acontecimiento, son subjetivas y, por ello, pueden ser erróneas, infundadas o desviadas, intervienen en el comportamiento de un individuo orientando su inserción o conocimiento del mundo. Estas también son socialmente compartidas, pasando algunas de ellas a través de generaciones familiares, sociales, culturales permitiendo, a su vez, dar cuenta las estructuras mentales de cada individuo.

Tal como se evidencia en las narrativas de los y las participantes, estos revelan las presunciones que cada uno tiene acerca del estudio y de sus proyecciones para el futuro, algunas son construidas de acuerdo a las experiencias de los sujetos en su cotidianidad, elaboraciones mentales que se hacen y que tienen como función facilitar la vida del mismo y otras que se hacen en conjunto con el otro y los otros, replicándose en sus contextos cotidianos.

Es notable como cada una de las personas significativas le dan importancia a la preparación académica, de cómo en cada una de sus narrativas exaltan virtudes del estudio como: acceso a: a) mejores oportunidades laborales, b) para ser alguien en la vida, c) para no sufrir, d) para mejorar expectativas frente al futuro, como se afirman a partir de las siguientes enunciados tomados de sus narrativas:

Persona significativa de $J E:$ “...... te da herramientas laborales, te da oportunidades laborales"

Persona significativa de $K$ : “... uno sin estudio no es nada, que le toca trabajar en cualquier cosa y eso si le resulta, que la cosa esta muy brava..."

8 Esta estructura perfectamente podría llamarse creencias; no obstante, con el ánimo de evitar malas interpretaciones y polisemias innecesarias en nuestro informe final, dado al uso terminológico y conceptual de estructuras de creencias, consideramos semántica y pragmáticamente posible utilizar el término presunciones. 
Persona significativa de $M A$ : “...es la base de todo, para mi concepto el estudio es muy importante, porque es la formación de cada persona y si uno quiere llegar a ser alguien en la vida uno tiene que prepararse"

Persona significativa de $E$ : “...pero me gustaría darle estudio a mi hija para que sea alguien en la vida, o sea, que no le pase lo que me paso a mí"

Persona significativa de $L$ : "...Porque es el camino hacia un mejor futuro", "... Porque yo quiero que ellos aprendan que se superen, que sean alguien en la vida, que no tengan que por decirlo así matarse tanto como a uno le toca...", "Entonces, eso es precisamente lo que uno no quiere para ellos, que ellos se queden ahí, dependiendo de un jornal por ahí; o sea, matándose al sol y todo. Que tengan un mejor futuro, algo que uno nunca tuvo"

Por consiguiente, se constata que las personas significativas a pesar de las diferencias de edades, ubicaciones geográficas, estratos socio-económicos presentan posturas muy similares frente al estudio, posición que van compartiendo en sus dinámicas familiares con sus hijos e hijas, las cuales estos a su vez las van adoptando y estructurándose así como presunciones.

En esta investigación, las presunciones se entienden como un estado que se encuentra dotado de contenidos representacionales que tiene un individuo acerca de todo aquello que lo acontece, las cuales son expresadas por los y las participantes como requerimientos o, quizá, como verdades establecidas, ya bien sea dentro del entorno familiar o social, dichas presunciones, se manifiestan como el deber ser o la forma pertinente de operar de una persona, tal como menciona el participante $L$

"Uno debe ser responsable con sus deberes pero no es sólo la obligación del hijo sino también de los padres, ellos también debe que ayudarnos en lo que necesite, siempre estar al lado apoyándonos y darnos ejemplo de lo que necesitemos".

En la narrativa se puede identificar la presunción que tiene el participante acerca de su percepción de la función y rol que deben ejercer su padre y su madre frente al proceso de desarrollo de sus hijos e hijas.

Desde estas narrativas se evidencia que una de las presunciones que 
se ha fundamentado en torno al estudio, está soportada en la siguiente expresión: "El estudio es la mejor herencia que un padre le puede dejar a un hijo". Esta idea se basa en un discurso social que ha imperado en el medio en los últimos 30 años aproximadamente, sobre lo mejor que puede hacer un padre y madre en pro de asegurarle el porvenir a su hijo e hija, es brindarle estudio para poder así, garantizarle un modo de supervivencia en su futuro. ${ }^{9}$

Por otro lado, otra de las presunciones que surgen en sus narrativas, es la idea de: $L$ : "Uno debe que estudiar para ser alguien en la vida para no tener que sufrir en la vida”, con esto, se valida la necesidad que tienen las personas de estudiar para poder así garantizarse un mejor futuro y no tener que sufrir necesidades económicas como lo han tenido que hacer sus cuidadores, al no tener estudio.

Lo anterior se sustenta bajo el imaginario que tienen varias de las personas significativas acerca del estudio como garante de mejorar principalmente las condiciones económicas, sin embargo, la estadísticas demuestran como los profesionales a pesar de su título no tienen solventadas estas condiciones.

Para finalizar, lo que se resalta en la mayoría de las narrativas revisadas que componen las narrativas de los y las adolescente sobre las presunciones que tienen del estudio, son visiones adulto céntricas retomadas básicamente de las de sus cuidadores o personas significativas y de los discursos sociales, pero no necesariamente presunciones que hayan reflexionado con base a sus necesidades y aspiraciones.

\section{Socialización}

Otra de las categorías emergentes hallada en la investigación fue la socialización y para Berger y Luckman (2003) la sociedad es entendida como un continuo proceso dialéctico conformado por tres momentos: la externalización, la objetivación y la internalización.

9 En la intención de voto para presidentes, las encuestas muestran que es más importante la educación que la paz para los votantes. 
Estos tres momentos caracterizan a la sociedad y, a la vez, a cada sector de ella. Estar en sociedad es ser partícipe de su dialéctica, aunque los sujetos no nacen miembros de una sociedad, sino que nacen con predisposición hacia la sociedad y, luego, llegan a ser miembros de la misma, participando entonces de su lógica. La participación es la socialización, la cual es definida como la introducción amplia y coherente de un sujeto en el entramado cultural de una sociedad o en un sector de él.

El proceso de socialización de un sujeto está compuesto por dos momentos, la primera, por la socialización primaria y la segunda, por la socialización secundaria. La socialización primaria es cuando el infante es recibido por una familia, construye su identidad a partir de otros significantes, en este caso personas de su núcleo familiar y posteriormente, se convierte en miembro de una sociedad. Luego, la socialización secundaria es cuando el sujeto ya socializado, participa de nuevos sectores de la sociedad, como son las instituciones educativas.

En la institución educativa, lo social se manifiesta como todo aquello que directa o indirectamente permea a los y las participantes en sus creencias y acciones de la vida diaria, que se construyen o surgen de la interacción con los otros y el otro.

Las narrativas de los y las adolescentes expresan dos puntos de vista, uno favorable y otro desfavorable. El favorable alude a lo motivante que resulta para ellos y ellas asistir a la institución educativa porque tienen amigos y amigas, que los animan a estar allí, los ayudan con sus quehaceres escolares y le trasmiten el gusto por el estudio.

Es la narrativa de $M A$ quién cuenta: "Los primeros años de la escuela lo disfrutaba porque estaba con mis compañeros" Y $A M$ quién dice: "Yo hice mi primaria aquí en la I.E Playa Rica e hice muchas amigas, pues yo soy muy amigable"

Estas narrativas presentan como el contacto con el otro en el espacio educativo fortalece el vínculo que se entreteje entre los y las participantes y sus compañeros y compañeras, haciéndole sentir motivación por asistir al colegio para entablar relaciones interpersonales, pues la 
institución no sólo se plantea aquí como un espacio rígido de aprendizajes con el docente, sino además, enriquecido por las relaciones establecidas entre los pares, como lo confirma la persona significativa de su entorno familiar. Persona significativa de $A M$ : “...yo creo que ella viene al colegio, porque acá tiene las amigas... se la pasa conversando en el salón de clase con las amiguitas..."

Por tanto, los amigos y las amigas se convierten en sujetos importantes que les permiten a los y las participantes cambiar su percepción sobre el estudio y fijar una meta con respecto a lo que quieren lograr estudiando, como es el caso de $L$ : "Al tiempo fui consiguiendo mejores amigos y ellos me ayudaron con los estudios y ahí fue donde comenzó a gustarme el estudio". Con la narrativa se identifica como la amistad, la solidaridad, la colaboración, el apoyo y el compañerismo generan en el adolescente otra perspectiva acerca del estudio, logrando que se sienta a gusto no sólo con sus compañeros y compañeras de escuela, sino además, que sienta agrado asistir a ella, ya que lo que le sucede a él se presentan como experiencias positivas.

Por otro lado, algunas vivencias que se generan al interior de la institución se tornan confusas, ya que las relaciones que se generan en aquellos lugares son significativamente negativas y lo que termina generando son resistencias por parte de los y las adolescentes para asistir a la institución y rendir académicamente. Tal situación es narrada por la siguiente participante:

$K$ : "Yo le decía a la profesora que no me sentía bien y ella me cogía todo duro y me pegaba con un libro en la cabeza y los compañeros me decían: itan boba!"

A partir de la anterior narración, se logra identificar que en lo social, ocasionalmente pueden surgir elementos que dificultan el arraigo de las participantes por la escuela.

Lo social para los y las adolescentes, se convierte en un factor importante que en cierta medida determina el amor por el estudio o la permanencia de ellos y ellas en las instituciones educativas. Francisco 
Cajiao (1995) dice: "El objetivo académico dista mucho de aprender, pues lo importante es pasar para mantener el cupo y los compañeros" (p.27). Es por ello, que los amigos y las amigas de la institución se convierten en el ancla que los mantiene unidos a la escuela, sin reconocer en algunas ocasiones los aprendizajes académicos que adquieren en estos espacios pero si las experiencias de vida que allí pueden tomar de la mano de sus pares.

En las anteriores narrativas, lo social se constituye como uno de los elementos que motiva a la permanencia de los y las adolescentes en la escuela, ya que se sienten reconocidos y apoyados por sus pares; sin embargo, surgen otras narrativas donde lo social en lugar de motivar crea resistencias, materializándose en algunos casos estas experiencias con un bajo rendimiento académico.

\section{Rendimiento académico}

El rendimiento académico es otra categoría emergente que surge en las narrativas y para Figueroa (2004, s.p), se define como el producto de la asimilación del contenido de los programas de estudio, expresado en calificaciones dentro de una escala convencional, es decir, el resultado cuantitativo que asigna cada docente a partir de la aplicación de pruebas o de otras actividades complementarias donde considera si el estudiante ha cumplido o no con el proceso de aprendizaje de conocimientos.

Por su parte, Aranda citado por Vigo Quiñones (2013, p.28) considera que es el resultado del aprovechamiento académico en función a diferentes objetivos y hay quienes homologan que el rendimiento académico puede ser definido como el éxito o fracaso en el estudio expresado a través de notas y calificativos.

Sin embargo, para el presente artículo, se entenderá el rendimiento académico como el proceso de evaluación, en el que las calificaciones escolares son el indicador cuantitativo que da cuenta del proceso de aprendizaje y el logro de objetivos establecidos. 
Ahora bien, el rendimiento académico es un fenómeno multifactorial, puesto que intervienen múltiples variables externas al estudiante, como el maestro, el contexto social, la familia, entre otras y variables internas, como la actitud, la inteligencia, la personalidad, la motivación, etc. En las narrativas de los y las adolescentes se hallan varias causas para el bajo rendimiento académico, el cambio de institución educativa, la actitud y la disposición del Otro.

De igual modo, se identifica que entre esas causas se destaca la falta de acompañamiento en la realización de tareas y demás trabajos por parte de los adultos y las adultos del hogar, la cual era visible en la primaria al apoyarlos en su elaboración, pero que al pasar de este ciclo escolar a la secundaria, se presenta un cambio abrupto disminuyendo el apoyo en el desarrollo de ellas, probablemente debido al desarrollo psicosocial que dichos participantes están viviendo, pues se provee que a mayor edad más responsabilidad. Esto se argumenta a partir de las siguientes narrativas, $M A$ "en la primaria me cuidaba una tía y mi prima me ayudaba a hacer las tareas, después cuando pasé a sexto grado ya nadie me ayudaba a hacer las tareas"... "En este momento hago las tareas solo, pero cuando estaba pequeño me acompañaba mi mamá y más pequeño, mi papá”.

Las narrativas expresadas por los y las participantes frente al acompañamiento que tienen sus personas significativas se confirman con las reproducidas por estos últimos: Persona significativa de $M A$ : “...seguimiento, seguimiento, se lo hice hasta quinto de primaria" "usted tiene una enciclopedia, internet, ahí tiene un libro, entonces no se excuse, si no entiende me dice".

Se identifica que en ocasiones el acompañamiento desde la presencia física se ha ido relevando, sustituyendo en este caso por herramientas tecnológicas, diferentes útiles escolares o ayudas didácticas, relegando de esta manera un poco su responsabilidad.

Lo que se evidencia aquí, es un llamado o manifestación de los y las adolescentes hacia los adultos y las adultas para que sigan acompañán- 
dolos y ayudándolos en la realización de las tareas y demás trabajos, ya que carecen de una figura de apoyo en los momentos de hacer las actividades extracurriculares.

Además de la falta de acompañamiento por parte de los adultos y las adultas del hogar, se encuentra el cambio de institución educativa, lo cual les marca otra exigencia en el desarrollo académico, los horarios, las metodologías y demás aspectos a los cuales se les dificultad adaptarse, como es el caso del siguiente participante:

JE: "En la parte académica si me fue un poco mal, desde cuarto grado venía con un bajón grande de notas, pero acá era más la exigencia académica"

JE: "Mayor impacto fue más que todo la... los procesos de enseñanza, porque en el tercero era más... por ejemplo acá son con libros con guías y yo no estaba acostumbrado a eso, yo estaba acostumbrado a que la profe nos enseñara y nos dictara, los proceso y los horarios, porque yo en el tercero estudiaba por la mañana"

Para los y las adolescentes que participaron en la investigación, estas causas se constituyen en un factor que ha incidido notablemente en su rendimiento académico, materializándose como tal en la reprobación del año escolar, en este caso grado sexto, incluso, el cual han perdido en una o dos ocasiones.

A lo largo de este artículo se han analizado algunas de las diferentes narrativas de los y las adolescentes y a la vez las de sus personas significativas sobre los que piensan y creen del estudio, surgiendo las siguientes categorías: los sentimientos, los vínculos, las actitudes, la sanción, las relaciones familiares, las presunciones, la socialización y el acompañamiento al proceso académico.

Recordando que el sentimiento son aquellas "emociones culturalmente codificadas, personalmente nombradas y que duran en el tiempo" (Fernández Poncela 2011, p.3), la culpa, es aquel sentimiento más recurrente en las narrativas de los y las adolescentes y personas significativas, puesto que los participantes no han cumplido con las res- 
ponsabilidades socialmente asignadas para poder obtener un buen rendimiento académico. Es decir, los y las adolescentes no retribuyen con su rendimiento los esfuerzos de sus personas significativas y estas últimas, no realizan un acompañamiento asertivo a los procesos escolares de sus hijos e hijas.

Por otro lado, el vínculo es aquel que hace referencia "al lazo afectivo que emergen entre dos personas y que genera un marco de confianza en el otro y en la vida, en un contexto de comunicación y de desarrollo”. (Bowlby, 1969, p.293), para lo cual, el vínculo más recurrente que han entablado los y las adolescentes en el escenario familiar ha sido con sus madres, las cuales se constituyen en las personas significativas de esta investigación. Las madres son personas significativas, porque son "aquellas personas importantes para el niño que tiene influencia en su desarrollo” (S.H Sullivan, 1953, como se citó en Domagala- Zyk, 2005, p.270), además son de las cuales los y las adolescentes retoman en parte las estructuras de creencias sobre el estudio.

La sanción es otra estructura de creencia que surgen en las narrativas en torno al estudio, ya que el y la adolescentes al no manifestar un buen rendimiento académico y tener comportamientos que perjudican la convivencia al interior de las aulas, son persuadidos por sus personas significativas con una serie de acciones y discursos con el fin de que reflexionen sobre lo que hacen o dejan de hacer en la escuela.

También en las narrativas de los y adolescentes surge la estructura de las relaciones familiares, pues al relatar sobre sus experiencias familiares surgen relatos en torno a relaciones difíciles y conflictivas entre las partes y estilos parentales que afectan de una u otra forma el rendimiento académico del adolescente, ya que como dice Vallejo Casarín y Mazadiego Infante (2006, p.56), "los estilos parentales inciden en el rendimiento académico de los y las adolescentes. Del estilo que se ejerza en cada hogar, autoritario, permisivo y democrático, dependerá directa o indirectamente el desempeño académico de los hijos e hijas en la escuela" en este caso, el estilos parental que más sobresale en las narrativas es el autoritativo. 
Por otro lado, entre las narrativas de los y las adolescentes se aprecia que la pereza es la actitud recurrente entre los y las adolescentes, además, ellos y ellas consideran que es principal motivo del bajo rendimiento académico, por esto, se identifica como una estructura de creencia, puesto que en el discurso de las personas significativas esta actitud es la causante de las dificultades académicas.

Fuera de lo anterior, en las narrativas se hallan una serie de presunciones, que al igual que las creencias son "productos o propiedades del pensamiento y, por lo tanto, también están asociadas con la mente. Aquí se entiende por creencia cualquier cosa que puede ser pensada" (Van Dijk, 2000, p.35), dichas presunciones tanto en adolescentes como en personas significativas aluden a tener mejores oportunidades laborales, ser alguien en la vida, no sufrir debido a las carencias económicas, mejorar las expectativas frente al futuro. Las anteriores presunciones son ideas que pasan de generación a generación, en este caso, pasan de las personas significativas a los y las adolescentes, y se conservan más desde una visión adultocentrica que desde la misma necesidad y deber ser del adolescente.

Otra de las estructura de creencias que hallan en las narrativas de los y las participantes, es la socialización, puesto que el estudio se convierte en la posibilidad que tienen la mayoría de los y las adolescentes para interactuar con otras personas y así entablar relaciones de amistad, confianza, solidaridad y apoyo generándose algo significativo para ellos y ellas. El estudio se convierte en lo que dice Francisco Cajiao (1995) "El objetivo académico dista mucho de aprender, pues lo importante es pasar para mantener el cupo y los compañeros” (p.27).

Finalmente, entre las narrativas surge en torno al estudio, la creencia del rendimiento académico, puesto que en los relatos de los y las adolescentes suscitan varios motivos para el bajo rendimiento académico, entre ellas, el cambio de institución educativa, la actitud y la disposición del otro. Así mismo, se identifica la falta de acompañamiento en la realización de tareas y demás trabajos por parte de los adultos y las adultos del hogar, la cual era visible en la primaria al apoyarlos en su elabora- 
ción, pero que al pasar de este ciclo escolar a la secundaria, se presenta un cambio abrupto disminuyendo el apoyo en el desarrollo de ellas, probablemente debido al desarrollo psicosocial que dichos participantes están viviendo, pues se provee que a mayor edad más responsabilidad.

Con respecto a todo lo anterior, algunas estructuras son construidas de acuerdo a las experiencias de los sujetos en su cotidianidad, a las elaboraciones mentales que hacen y que tienen como función facilitar la vida del mismo y otras que se hacen en conjunto con el otro y los otros, replicándose en sus contextos cotidianos.

Con las estructuras que surgen en esta investigación se comprende en medida el porqué del bajo rendimiento académico de los y las estudiantes del grado sexto, manifestándose una serie de creencias que influyen considerablemente en este proceso escolar de los y las adolescentes, las cuales han sido retomadas de sus personas significativas y del medio social y cultural en los que se encuentran inmersos.

\section{Conclusiones}

El articulo expone los resultados que se tejen en torno a la investigación "Relación entre las estructuras de creencias de los y las adolescentes con bajo rendimiento académico con las de sus personas significativas" develando que las estructuras de creencias que son trasmitidas por las personas significativas a los adolescentes en su cotidianidad y experiencia acerca del estudio por medio de acciones y discursos, hacen referencia a tener mejores oportunidades laborales, a ser alguien en la vida, a no sufrir y mejorar las expectativas frente al futuro.

Se deja entrever, que de acuerdo a estas creencias, las personas significativas han ido construyendo un discurso que día a día trasmiten a sus hijos o hijas pero que no se materializa, porque sus acciones no son coherentes con sus palabras, es decir, con sus ejemplos de vida, no logran impactar a los y las adolescentes, ya que muchas de esas personas no han terminado sus estudios básicos, ni tampoco manifiestan un 
verdadero interés por seguir formándose académicamente, además no apoyan a sus hijos e hijas en la elaboración de deberes escolares.

También con la investigación se puede entrever que muchas de las creencias que tienen los y las adolescentes acerca del estudio, son visiones adultocéntricas que son retomadas básicamente de las de sus cuidadores o personas significativas y de los discursos sociales, pero no necesariamente presunciones que hayan reflexionado con base a sus necesidades y aspiraciones.

Finalmente, cabe anotar que al identificar y describir las narrativas de los y las adolescentes y las encuestas sociodemográficas y las entrevistas de sus personas significativas, se comparan, estableciendo las siguientes semejanzas y diferencias:

Tabla N 1. Semejanzas entre los y las adolescentes con sus personas significativas.

\section{Semejanzas}

\section{Adolescentes}

Se logra identificar que los y las adolescentes a pesar de su bajo rendimiento académico aspiran terminar sus estudios de secundaria.

\section{Personas significativas}

Las personas significativas que participaron en la investigación apoyan desde el discurso a los y las adolescentes para que culminen sus estudios de secundaria. Tal como se evidencia en el siguiente relato: MA: "Mi mamá es la persona más significativa que he tenido durante el proceso educativo porque ella me apoya"

Para los y las adolescentes el estudio es el medio para el logro de muchas metas: "salir adelante" y mejorar su calidad de vida.
De igual forma, en el discurso de las personas significativas es recurrente escuchar que el estudio es el medio para "Salir adelante". 
Uno de los sentimientos más recurrentes de los y las adolescentes en sus narrativas es la culpa, por el bajo rendimiento académico que evidencian periodo tras periodo y que no cumple con las expectativas de sus personas significativas.
Así mismo, las personas significativas también manifiestan en sus narrativas culpa por el bajo rendimiento académico, ya que sienten responsables no realizar un acompañamiento asertivo a los procesos educativos de sus hijos. Lo anterior se soporta en el siguiente relato:

Personas significativas de MA: "entonces a mí me duele mucho que yo no he estado con él, me refiero a que lo despache, que lo lleve, que tan rico como mamá poderlo estar aquí pendiente cuando llegue, se va, darle la comida, el transporte...eso me duele mucho"

Los adolescentes reconocen la importancia de estudiar pero no realizan acciones coherentes con lo que dicen.
Las personas significativas también evidencian incongruencia entre lo que dicen y hacen, puesto que reconocen la importancia del estudio, pero no realizan un acompañamiento asertivo en el proceso educativo de sus hijos.

El discurso de los y las adolescentes acerca del estudio es similar al de sus personas significativas, Las personas significativas ven en el estudio beneficios para la vida, replicando esta estructura constantemente en sus discursos.

puesto que ven los mismos beneficios en el estudio que sus personas significativas.

Fuente: Propia

Teniendo en cuenta las anteriores semejanzas entre las creencias de los y las adolescentes y las de sus personas significativas, se puede aseverar que hay una incidencia a partir del manejo del discurso de las personas significativas a los y las adolescentes sobre los beneficios que tiene para la vida el hecho de estudiar.

Es importante reconocer que debido al proceso de socialización primaria que exponen Berger \& Luckmann (2003), las estructuras de creencia de los y las adolescentes se retoman de sus personas significativas, puesto que ellos han ido adoptando ciertas posturas que se tras- 
miten en el discurso social, aunque en algunas ocasiones se transforman en su socialización secundaria, sin embargo, en esta investigación es recurrente escuchar en sus narrativas discursos compartidos entre adolescentes y personas significativas sobre el estudio.

Tabla N 2. Diferencias entre los y las adolescentes con sus personas significativas.

\begin{tabular}{|c|c|}
\hline \multicolumn{2}{|c|}{ Diferencias } \\
\hline Adolescentes & Personas significativas \\
\hline $\begin{array}{l}\text { La sanción como la consecuencia que } \\
\text { no rendir académicamente. }\end{array}$ & $\begin{array}{l}\text { La sanción es empleada como ejer- } \\
\text { cicio de autoridad, buscando a par- } \\
\text { tir de esta corregir y sensibilizarlos } \\
\text { acerca de sus acciones. }\end{array}$ \\
\hline $\begin{array}{l}\text { Los y las adolescentes manifiestan en } \\
\text { sus narrativas actitudes de desinte- } \\
\text { rés y negligencia frente a las respon- } \\
\text { sabilidades académicas. } \\
\text { Lo anterior se argumenta en la si- } \\
\text { guiente narrativa: } \\
\text { JE: "Umm... bueno di cierto bajón } \\
\text { por el cambio de tema y más estricto } \\
\text { y más... como decirlo y más, si más } \\
\text { estricto y temas más difíciles y cier- } \\
\text { ta pereza y como se dice... umm cier- } \\
\text { to bajón de actitud por parte mía del } \\
\text { estudio, pues me dejo de importar } \\
\text { y... yo creo que eso fue la base de } \\
\text { todo de la pérdida del año". }\end{array}$ & $\begin{array}{l}\text { Por otro lado, las personas significa- } \\
\text { tivas asumen una actitud impositiva } \\
\text { para que los adolescentes cumplan } \\
\text { con sus responsabilidades. }\end{array}$ \\
\hline
\end{tabular}


Los y las adolescentes manifiestan Por otro lado, las personas significaque su bajo rendimiento académico tivas expresan que ellos hicieron el obedece en cierta medida a la falta acompañamiento solamente hasta de acompañamiento por parte de sus quinto de primaria, que en bachipersonas significativas. llerato es asunto de los adolescentes responder por su rendimiento.

Persona significativa de MA: “... porque me cansé honestamente yo me cansé, el seguimiento que yo le hubiera hecho a Miguel, seguimiento, seguimiento, se lo hice hasta quinto de primaria...".

Fuente: Propia

Por otro lado, las diferencias que se evidencian en las narrativas de los y las participantes de esta investigación dejan entrever que el bajo rendimiento académico es atribuido en cierta parte al otro, puesto que no aceptan que es una responsabilidad compartida, donde el adulto no cumple con el acompañamiento en la elaboración de deberes escolares y el estudiante por su actitud de pereza no rinden académicamente.

\section{Referencias}

Berger, P.L., y Luckmann, T. (2003). La construcción social de la realidad. Buenos Aires: Amorrortu editores.

Bolwby, J. (1969). El vínculo afectivo. Buenos Aires. Ed. Paidós.

Cajiao, F. (1995). La adolescencia en el universo de las edades de la vida. En: FES.

(Ed.). Proyecto Atlántida: estudio sobre el adolescente escolar en Colombia (pp.1-50). Bogotá.

Fernández, A. M. (2011). Antropología de las emociones y teoría de los sentimientos. Revista Versión Nueva Época (26), p.1-24. Recuperado de http://148.206.107.15/biblioteca_digital/artículos/7-552-80580sn.pdf 
Figueroa, C. (2004). Sistemas de evaluación académica. San Salvador: Editorial Universitaria.

Hernando, A., Oliva, A., y Pertegal, M.A, (2012). Variables familiares y rendimiento académico en la adolescencia. Estudios de Psicología, 33(1).

Londoño, D. (2015). Jóvenes y literacidad: Un análisis sociolingüístico. Envigado: Sello IUE.

Rossas Sosa, L.M (1997). Funcionamiento familiar y rendimiento escolar [Tesis de maestría]. Universidad Autónoma de Nuevo León. Nuevo León. Recuperado de http://cdigital.dgb.uanl.mx/te/1080o80898.pdf

Sánchez, P. (s.f). Caracterización de los alumnos de alto riesgo de fracaso escolar en el COBACH. Universidad Autónoma de Yucatán. Recuperado de http://www. cpti.com.mx/riesgo\%20alumnos.pdf

Secretaria de Educación Distrital de Bogotá (2010). Estudio sobre los factores que influyen en el rendimiento escolar. Recuperado de http://evaluacion.educacionbogota.edu.co/files/Factores\%20que\%20influyen\%20en\%20el\%20rendimiento\%20escolar.pdf

Tilano, L.M.; Henao, G.C., y Restrepo. J.A. (2009). Prácticas educativas familiares y desempeño académico en adolescentes escolarizados en el grado noveno de instituciones educativas oficiales del municipio de Envigado. 9(1). Recuperado de http://web.usbmed.edu.co/usbmed/elagora/htm/v9nro1/ pdf/cap2.pdf

Vallejo, A., y Mazadiego, T.J. (2006). Familia y rendimiento académico. Revista de Educación y Desarrollo, 5, 55-59.

Van Dijk, T. (2000). Ideología, un enfoque multidisciplinario. Barcelona: Gedisa. Vigo Quiñones, A. (s.f). Influencia de los hábitos de estudio en el rendimiento académico de los estudiantes del I año del ISTH - Huaral. Recuperado de http:// 
Diana Cecilia Pérez Posada / Fray Durley Arrieta Osorio / Jesús Quintana Castillo / David Alberto Londoño Vásquez

www.monografias.com/trabajos-pdf4/habitos-estudio-rendimiento-academico- estudiantes-del-isth/habitos-estudio-rendimiento-academico-estudiantes-del-isth.pdf 